\title{
Total-Body PET: Maximizing Sensitivity to Create New Opportunities for Clinical Research and Patient Care
}

\author{
Simon R. Cherry ${ }^{1,2}$, Terry Jones ${ }^{2}$, Joel S. Karp ${ }^{3}$, Jinyi Qi ${ }^{1}$, William W. Moses ${ }^{4}$, and Ramsey D. Badawi ${ }^{1,2}$ \\ ${ }^{1}$ Department of Biomedical Engineering, University of California, Davis, California; ${ }^{2}$ Department of Radiology, University of \\ California Davis Medical Center, Sacramento, California; ${ }^{3}$ Department of Radiology, University of Pennsylvania, Philadelphia, \\ Pennsylvania; and ${ }^{4}$ Lawrence Berkeley National Laboratory, Berkeley, California
}

PET is widely considered the most sensitive technique available for noninvasively studying physiology, metabolism, and molecular pathways in the living human being. However, the utility of PET, being a photon-deficient modality, remains constrained by factors including low signal-to-noise ratio, long imaging times, and concerns about radiation dose. Two developments offer the potential to dramatically increase the effective sensitivity of PET. First by increasing the geometric coverage to encompass the entire body, sensitivity can be increased by a factor of about 40 for total-body imaging or a factor of about 4-5 for imaging a single organ such as the brain or heart. The world's first total-body PET/CT scanner is currently under construction to demonstrate how this step change in sensitivity affects the way PET is used both in clinical research and in patient care. Second, there is the future prospect of significant improvements in timing resolution that could lead to further effective sensitivity gains. When combined with total-body PET, this could produce overall sensitivity gains of more than 2 orders of magnitude compared with existing state-of-the-art systems. In this article, we discuss the benefits of increasing body coverage, describe our efforts to develop a first-generation total-body PET/CT scanner, discuss selected application areas for total-body PET, and project the impact of further improvements in time-of-flight PET.

Key Words: instrumentation; molecular imaging; PET/CT; instrumentation; PET; total-body imaging

J Nucl Med 2018; 59:3-12

DOI: 10.2967/jnumed.116.184028

A 11 nuclear medicine studies in humans are limited by the trade-offs between the number of detected decay events, imaging time, and absorbed dose. The number of detected events determines the signal-to-noise ratio (SNR) in the final image, but constraints on administered activity, as well as high random event rates and dead time that occur at high activities, currently prevent acquisition of high-SNR images in short times. This in turn limits the ability to perform high-resolution, dynamic imaging studies with tracer kinetic modeling, because short-time-frame datasets are always noisy. A further limitation is that although the tracer injection is systemic and radiotracer is present in the entire body,

Received Jul. 3, 2017; revision accepted Sep. 11, 2017.

For correspondence or reprints contact: Simon R. Cherry, Department of Biomedical Engineering, University of California, Davis, One Shields Ave., Davis, CA 95616.

E-mail: srcherry@ucdavis.edu

Published online Sep. 21, 2017.

COPYRIGHT (c) 2018 by the Society of Nuclear Medicine and Molecular Imaging. current imaging systems contain only a small portion of the body within the field of view (FOV). For applications in which the distribution of radiotracer in the entire body or multiple organ systems is of interest, this limitation leads to further inefficiencies and makes it difficult to acquire dynamic data from all the tissues of interest. If one takes whole-body PET scanning with ${ }^{18} \mathrm{~F}-\mathrm{FDG}$ as an example, the total efficiency with which pairs of coincidence photons that escape the body are detected is well under $1 \%$ even on today's best scanners. Simplistically, this number can be derived by considering that the average geometric sensitivity within the FOV of a typical clinical PET scanner is under 5\% and that with an axial coverage of $20 \mathrm{~cm}$, less than an eighth of the body is in the FOV at any one time. In this article, we discuss how improving the geometric coverage directly increases sensitivity by over an order of magnitude for total-body PET scans. We describe progress to date on building the first prototype total-body scanner that will show a roughly 40 -fold sensitivity gain over existing PET scanners for imaging the entire body. We also discuss how such a large increase in effective sensitivity has the potential to dramatically broaden the utility of PET in human medical research, potentially leading to new clinical applications, as well as how it can positively affect existing clinical applications. Finally, we describe how anticipated improvements in timing resolution can produce further increases in effective sensitivity, leading to the prospect of PET scanners that are about 200 times more sensitive than current commercial systems for total-body imaging.

\section{FROM WHOLE-BODY PET TO TOTAL-BODY PET}

There are two major factors contributing to the extremely poor sensitivity $(<1 \%)$ for current whole-body PET scans (Fig. 1A). The first and most obvious is that at any one time, roughly $85 \%-$ $90 \%$ of the body is outside the FOV of the scanner and no signal from these regions of the body is collected. Second, even for the tissues and organs within the scanner FOV, no more than about $3 \%-5 \%$ of the available signal (photon pairs that escape the body without being attenuated or scattered) can be collected, because the radiation is emitted isotopically and most does not intercept the detector rings. Both factors are addressed by extending the detector rings so that they cover the entire body (Fig. 1B). This concept, which we call total-body PET, allows almost maximal detection of the radiation emitted from the body. Computer simulations of a cylindric phantom approximating the dimensions of the adult human predict that extending a PET scanner from a typical $20-\mathrm{cm}$ axial FOV to a $200-\mathrm{cm}$ FOV leads to an approximately 40 -fold increase in the effective sensitivity (as measured by the noise-equivalent counting rate) for imaging the body from 


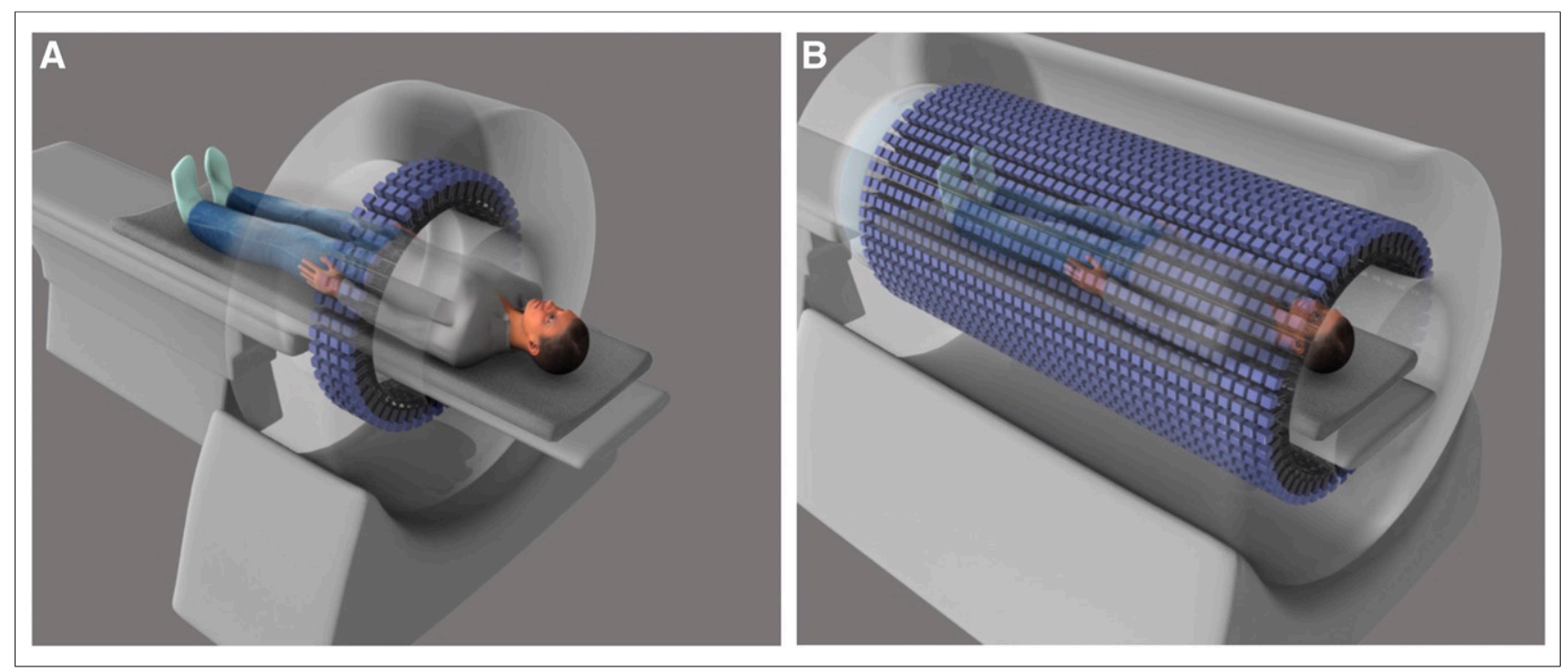

FIGURE 1. Whole-body PET (A) vs. total-body PET (B). (Reprinted with permission of (31).)

head to toe $(1,2)$. The major application in which the entire body is imaged by PET is melanoma. More commonly in clinical scans, the coverage is from "eyes to thighs," with the predicted sensitivity gain being about 24 -fold. The gains for imaging a single organ (e.g., brain or heart) are more modest because these organs already fit within the FOV of existing scanners. However, even here, the second factor is important and leads to a 4- to 5-fold increase in effective sensitivity.

\section{Impact}

The predicted 40-fold gain in sensitivity for a total-body PET scanner has major implications for molecular imaging. For a given scanning situation, the SNR in reconstructed PET images is dominated by the Poisson statistics inherent in radionuclide decay detection and is proportional to the square root of the number of detected events, $N$. This in turn depends on the injected activity, $A$, and the imaging time, $T$. To first order, the SNR in a reconstructed PET image can be approximated by

$$
\mathrm{SNR} \approx k \sqrt{S \times A \times T},
$$

where $k$ is a constant and $S$ is the effective sensitivity of the scanner. If $S$ is increased by a factor of 40 , several consequences are apparent. First, the sensitivity gain can be used to increase the

\section{NOTEWORTHY}

- The concept of total-body PET refers to a scanner that encompasses the entire body within the FOV and allows imaging of all the tissues and organs of the body simultaneously.

- The increase in geometric coverage of total-body PET produces a sensitivity increase of a factor of about 40 for imaging the entire body.

- Total-body PET images could be obtained with 6-fold better SNR, or in $1 / 40$ the scanning time or with $1 / 40$ the injected activity while maintaining existing SNR.

- The world's first total-body PET scanner, called EXPLORER, is currently being constructed.
SNR in the reconstructed image by a factor of $\sqrt{ } 40=6.3$ using the same protocols (injected activity, imaging time) as those we currently use. This gain could support reconstructing images at higher spatial resolution and would presumably allow detection of smaller or lower-contrast structures and lesions and improved quantification in both static and dynamic scans.

Second, one could choose to use the sensitivity gain to reduce imaging times by a factor of 40 while maintaining the SNR at its current level. Thus a 10- to 20-min total-body protocol could be completed in just 15-30 s! One could argue under these circumstances that there could also be additional improvements in image quality, because there would be far less patient motion, and singlebreath-hold PET imaging would be feasible.

Finally, the sensitivity gain could be used to reduce the injected activity by a factor of 40 . Thus, total-body ${ }^{18} \mathrm{~F}$-FDG scans could be performed by injecting just $9.25 \mathrm{MBq}(250 \mu \mathrm{Ci})$ rather than $370 \mathrm{MBq}(10 \mathrm{mCi})$ with the same SNR as is currently achieved (SNR may actually improve because of the reduced randoms fraction and dead time at lower activities). This dose reduction would allow PET scanning at effective doses of less than $0.2 \mathrm{mSv}$, potentially opening broader use of PET in sensitive populations (infants, children, and adolescents) and allowing up to 40 scans of a subject for the same effective dose as currently received in a single scan.

A less obvious consequence is that the increase in SNR broadens the dynamic range of the scanner and allows radiotracers to be followed for a longer time before the signal decays to such an extent that it is not detectable. A 40-fold increase in sensitivity means that a tracer could be followed for 5-6 additional half-lives (ignoring biologic clearance) above the approximately 3 half-lives possible on existing scanners. For labeled radiotracers, the implication is that imaging could be conducted as late as about $18 \mathrm{~h}$ for ${ }^{18} \mathrm{~F}, 3 \mathrm{~h}$ for ${ }^{11} \mathrm{C}$, and $18 \mathrm{~min}$ for ${ }^{15} \mathrm{O}$. For a long-lived radiotracer such as ${ }^{89} \mathrm{Zr}$, sufficient signal might still be available for imaging about $30 \mathrm{~d}$ after injection. The wide dynamic range also improves the viability of multitracer studies, in which a first tracer is injected at say $1 / 20$ the activity of a second tracer introduced a few minutes later, such that the signal from the first tracer would only minimally interfere with that from the second tracer. 
Last, a critical advantage of a total-body PET scanner is that the entire body is in the FOV at one time and every tissue and organ can be imaged simultaneously. This presents interesting opportunities for studying the body as a system and using systems kinetic modeling approaches (3). Total-body dynamic imaging (4,5), which to date has been hampered by poor SNR and sparse temporal sampling due to the need for multiple bed positions, will be much more practical, and the high SNR means that even data from very short frames will carry high-quality information for kinetic analysis. Furthermore, the heart chambers and major blood vessels will always be in the FOV, enabling high-statistics image-derived input functions to be obtained for total-body parametric imaging.

\section{TECHNOLOGIC DEVELOPMENT AND CHALLENGES}

\section{History}

The concept of PET scanners with a long axial FOV is not new, with a documented history of groups and individuals proposing and simulating such systems (6-11). There has been a slow, but inexorable, move to increase the axial length of commercial PET systems from the $10 \mathrm{~cm}$ common in the early 1990 s to the 20$30 \mathrm{~cm}$ used in most systems today; however, they still cover only a fraction of the body. The longest-axial-FOV scanner built to date was a $68.5-\mathrm{cm}$-long research scanner developed by Hamamatsu (12). However, the slow and relatively low-light-yield scintillator (bismuth germanate) necessitated the use of coarse axial septa to minimize scatter and counting rate problems, greatly reducing the possible sensitivity gain. There also has been a range of computer simulation studies designed to examine the effects of longer axial FOVs and the impact on scatter and random coincidences, as well as to determine the most effective way to distribute a fixed volume of scintillator material given this is a key determinant in the overall cost of a PET system $(1,7,10,13-17)$. The availability now of improved scintillation materials based on lutetium compounds, which have a more favorable combination of speed, light output, and stopping power, has made it feasible to develop fully 3-dimensional systems that can handle the higher counting rates and scatter fraction, and additionally provide time-of-flight information to further improve performance.

\section{Challenges}

Developing a total-body PET scanner at this point is largely an engineering and economic challenge rather than a scientific challenge, although there are many difficult design choices that require optimized solutions. Existing time-of-flight PET detector technology used in current-generation PET/CT and PET/MRI scanners can in principle be used. Although it would be desirable to further improve timing resolution, and incorporate depth-ofinteraction encoding into the detectors to account for increased parallax errors caused by the long cylindric geometry (18), the largest increment in sensitivity and utility comes immediately from the geometry of the total-body PET concept, which covers the entire body and efficiently detects almost all the photons that leave the body, regardless of the direction in which they are emitted.

The first significant challenge relates to the event rates and system electronics. The rate at which single events are detected by each detector module is in fact no higher than in current systems for the same injected activity. The challenges therefore are at the coincidence electronics, which must process data from many more detectors and will have to, in real time, handle far higher rates of singles and coincidence events. An alternative approach is to extract coincidences in software, after data have been collected. In this scenario, each detector module streams single events to disk with a precise and accurate time stamp, and coincidence events between modules are formed by sorting through all the singles data later. This design removes the data collection bottlenecks, as data can be streamed onto multiple disks in parallel to support the event rate, if the data across all hard drives are properly synchronized in time. This singles-based acquisition approach also has the advantage that timing and energy windows or weighting can be applied on an event-by-event basis during reconstruction and can be varied after data are collected for optimal image quality for any given application. For example, applying a variable coincidence timing window, based on the known body contour (extracted from the CT scan) and length of a line of response through the body, will reduce the contribution of random events (19).

The second challenge relates to how large amounts of data (listmode datasets could easily contain tens of events or even a hundred billion events and reach $>1 \mathrm{~TB}$ in size) are moved around, processed, and reconstructed in a timely fashion. This challenge requires appropriate thought and investment in the computational infrastructure of the scanner to ensure that these steps do not limit the use and number of subjects per day that can be scanned. Ideally, the processing time for each study should be on the order of the acquisition time so that in the worst case, all data processing can happen overnight to process the previous day's acquisitions. With the need to reconstruct approximately 40-fold more events for a standard injected activity, and the large size of the image matrix given the large axial FOV, image reconstruction is likely to be the computational bottleneck. However, given the parallel nature of the problem, it is largely a question of providing sufficient computing power and memory, which is well within the capability of existing technology assuming that the computational infrastructure is properly resourced.

The third challenge relates to mechanical design and engineering. Detector modules need to be packed tightly together in both axial and transaxial directions because any significant gaps quickly reduce sensitivity. At the same time, modules need to have excellent temperature stability, with cooling to handle the heat generated by the electronics, and must be accessible for servicing or replacement. These are perhaps some of the most difficult engineering challenges in realizing a high-sensitivity, reliable, and robust scanner. There also are additional mechanical design issues related to the long cantilever of the bed and the need for internal bore covers that are strong, low-attenuation, liquidimpermeable, and easily cleaned or sterilized.

The economic challenge will be an obvious one to the reader. What is the cost of such a device going to be? With health-care costs increasing, how can such an expensive device be justified? Presently, this question can only be addressed in part, because what ultimately matters is the cost-to-benefit ratio, and the benefit cannot begin to be quantified until the first scanners are built and operational. Although a total-body PET scanner is roughly equivalent to 8 conventional PET scanners, the cost of a total-body scanner is not expected to scale by a factor of 8 (because of the CT and other components that are common, and some economies of scale). Rather, initial estimates from industry suggest that a price tag of around \$10 million for a total-body PET/CT scanner is feasible, compared with $\$ 1.5$ to $\$ 2$ million for a standard PET/CT scanner. Given that the cost of the instrument is only one component of the cost of performing a clinical PET scan, and that a total-body PET/CT scanner could do the workload of several PET scanners without the corresponding increase in technical staff and 
expensive hospital real estate, it is not impossible, even at this price point, that an economic justification can be made. A further impact on the cost infrastructure of PET is that the increased sensitivity of total-body PET permits studies to be performed using a much lower injected activity, thus allowing radiopharmaceutical distributors to deliver clinically relevant doses over much longer distances or produce many more doses per synthesis.

In the research environment, where we envision the main initial uptake of such a scanner would be, the considerations regarding cost are quite different. The ability to acquire unique kinetic data with a total-body scanner system, and conduct research that directly converges with the growing focus on systems medicine, is hard to put a price on. Perhaps a reasonable analogy is to look at the evolution of MRI systems. The cost for a human 7-T MRI scanner, with building and installation costs, exceeds the estimated $\$ 10$ million for a total-body PET/CT scanner. Yet, there are about 50 such installations in the world already. And one can argue that the incremental performance advantage in moving from 3 to $7 \mathrm{~T}$, and its impact on applications, may turn out to be at best comparable, and perhaps inferior, to the potential step-change in both performance and total-body imaging coverage provided by the total-body PET concept. Time will tell.

\section{Multimodal Integration with CT or MRI}

Total-body PET will also need integration with an anatomic imaging modality. From a workflow and cost perspective, CT is the most attractive starting point. Total-body CT scans can be acquired rapidly (potentially as the subject is being moved into the PET scanner), and a standard CT scanner can be used in the conventional tandem PET/CT configuration. The one major limitation of CT in this context is the additional radiation dose, which will be a factor when using the low-dose capability of totalbody PET. However, there has been significant progress in lowdose CT protocols with advanced reconstruction methodology (20), and for diagnostic use and many research applications the effective dose using these techniques is likely acceptable. When the CT is needed only for attenuation correction, further reductions in radiation dose are possible, as the spatial resolution and signal-to-noise requirements on the CT are somewhat relaxed. For applications in which only extremely low levels of absorbed dose can be tolerated, there are other opportunities for deriving attenuation correction factors, either by applying the natural background radioactivity present in the lutetium-based scintillators used in most PET scanners (21) or by introducing a small number of external line sources as transmission sources (22). When combined with time-of-flight information that can aid in separating events that arise from the radiotracer within the body from events arising from transmission sources outside the body, and advanced joint estimation reconstruction methods, very-low-dose simultaneous emission-transmission imaging may be feasible.

Although integration with total-body MRI may be technically feasible, the fact that rapid total-body MRI protocols (23) are not widely used and require movement of the subject through the relatively small homogeneous-field region used for imaging, not to mention the costs and engineering challenges that would be associated with such a development, suggest it is prudent to start total-body PET developments using CT as the anatomic imaging modality.

\section{REALIZING TOTAL-BODY PET}

After 6 y of preparatory work, in 2011 we were awarded a grant from the National Cancer Institute as part of their Provocative
Questions Initiative to undertake detailed feasibility studies of total-body PET. We formed the EXPLORER consortium (24), led by the University of California, Davis, in collaboration with research groups at the University of Pennsylvania and the Lawrence Berkeley National Laboratory, to tackle this project and over the course of the last several years also have greatly benefited from the input of expert researchers and clinicians around the world. In late 2015, we received a Transformative Research Award (R01) from the National Institutes of Health under their High-Risk, HighReward Research Program (25) to build the world's first totalbody PET/CT scanner. Significant progress now has been made toward the first prototype, and critically, there is now engagement with industry and the growing interest from within the molecular imaging community that highly increases the likelihood that totalbody PET/CT will become commercially available. In this section, we summarize progress to date and describe the design of the first prototype system.

\section{EXPLORER Mock-up}

In collaboration with United Imaging Healthcare America (Houston, TX) we have developed a mock-up of the human EXPLORER scanner (Fig. 2) that allows us to study a range of practical issues related to the geometry of the scanner. The system consists of the gantry and bore covers, as well as the patienthandling system. With this mock-up, we plan to study patient tolerance of different scanning protocols, especially whether claustrophobia is an issue, and evaluate methods to reduce anxiety and motion using images projected above the patient's face, air flow through the bore, and other approaches. We will investigate motion-tracking strategies using cameras (both to evaluate how to minimize motion and ultimately to apply correction for motion during imaging) and using bed deflection and correction. We also will study challenges associated with injecting tracers for dynamic imaging deep inside the bore, as well as access for arterial blood sampling. Finally, we will use the mock-up to examine work flow and personnel requirements for different protocols, especially long dynamic scans with blood sampling and metabolite analysis. Once the actual EXPLORER scanner is built, we plan to retain this mockup to use as a simulator for subject training, thus allowing subjects to gain experience and become more comfortable with the scanning environment without affecting scheduling on the EXPLORER scanner itself. Such practice on the mock-up is likely to improve patient enrollment and compliance with imaging protocols for critical studies.

\section{EXPLORER Total-Body PET/CT Scanner Design}

The design of the first prototype EXPLORER scanner in collaboration with United Imaging Healthcare has been completed (Fig. 3), and the components are being fabricated and tested. The detector modules are based on $2.76 \times 2.76 \times 18.1 \mathrm{~mm}$ lutetiumyttrium oxyorthosilicate scintillator crystals read out by silicon photomultipliers. On the basis of preliminary measurements, these detectors exhibit an energy resolution of about $12.5 \%$ and a timing resolution of about $400 \mathrm{ps}$. These detector modules will be assembled to form a scanner with a diameter of $78.6 \mathrm{~cm}$ (clear bore, 70 $\mathrm{cm}$ ) and an axial length of $195 \mathrm{~cm}$. There will be a total of 564,480 lutetium-yttrium oxyorthosilicate crystals and 53,760 silicon photomultipliers in the system. A 64-slice CT scanner will be mounted on the front of the system for anatomic imaging and to provide the necessary information for attenuation correction. The footprint of the system (not including bed) is expected to be 290 $\mathrm{cm}$ long by $191 \mathrm{~cm}$ wide and-when accounting for the required 


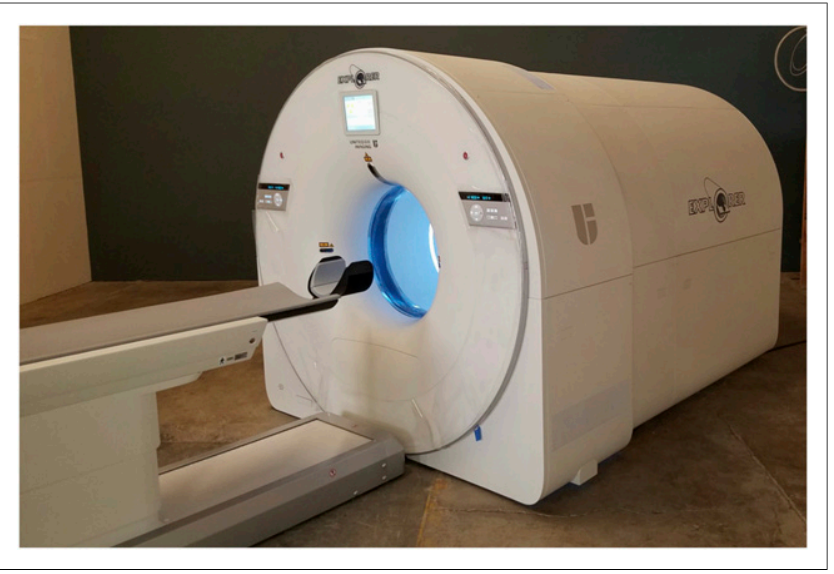

FIGURE 2. Photograph of EXPLORER mock-up installed at University of California Davis Medical Center.

travel of the bed and the space needed to separate detector rings for servicing - can be accommodated in a room that is $4-5 \mathrm{~m}$ wide by $8-9 \mathrm{~m}$ long, which is not significantly greater than the room sizes used in most current PET/CT and PET/MRI installations. Both real-time coincidence sorting (up to acceptance angles of $\pm 57^{\circ}$ ) and the option to stream all single events to disk and form coincidences off line will be available. The expected effective sensitivity gain of this first prototype for an adult subject, relative to a Siemens mCT PET/CT scanner (26), which was used for baseline computer modeling and simulations, is anticipated to be between 30 and 50, with the exact number depending on the timing resolution that is achieved at the system level. Construction of the system is expected to be completed in the third quarter of 2018, with the first imaging studies commencing shortly thereafter.

Concurrently, the University of Pennsylvania is designing a second prototype total-body system based on digital silicon photomultipliers $(27,28)$ to evaluate the benefits of even better timing resolution in the total-body geometry. The detectors, which are based on those in the Philips Vereos PET/CT scanner, use $8 \times 8$ arrays of $3.9 \times 3.9 \times 19 \mathrm{~mm}$ lutetium-yttrium oxyorthosilicate scintillation crystals, coupled in a 1-to-1 configuration to the digital silicon photomultiplier sensor. Compared with the commercial implementation with these detectors, which achieves a timing resolution of $320 \mathrm{ps}(29)$, the total-body prototype will operate at a lower temperature $\left(5^{\circ} \mathrm{C}-10^{\circ} \mathrm{C}\right)$ to achieve a timing resolution of below $250 \mathrm{ps}$. A prototype detector ring with a transverse diameter of $76.4 \mathrm{~cm}$ and an axial extent of $22.9 \mathrm{~cm}$ has been constructed and will be expanded to a larger axial FOV by adding further rings.

\section{Mini EXPLORER}

We also have developed a small-scale mini EXPLORER scanner designed to recapitulate some of the large axial acceptance angles of a total-body scanner but on a more manageable scale that is appropriate for total-body PET imaging in nonhuman primates (rhesus macaques). This system allows the effect of collecting axially oblique lines of response (e.g., trade-offs between sensitivity, resolution, scatter fraction, and randoms fraction) to be determined, as well as providing a platform for developing data correction and image reconstruction strategies. However, it has a second more important purpose, and that is to provide a fully functional system that can be used to develop and evaluate some of the more speculative or early-stage application ideas in nonhuman primates before use in human subjects. This purpose takes advantage of the fact that the University of California Davis is home to one of the 7 National Institutes of Health-funded primate centers and that the mini EXPLORER will be housed alongside an existing clinical PET/CT scanner in that facility.

The mini EXPLORER system is based on the components from a Siemens mCT scanner (26), which have been reconfigured into a scanner that is roughly half the diameter and twice the axial length. The result is a system with a bore diameter of $43.5 \mathrm{~cm}$, a transaxial FOV of $30 \mathrm{~cm}$, and an axial FOV of $45.7 \mathrm{~cm}$. Performance characterization shows a sensitivity gain of 5-fold over the standard mCT using the National Electrical Manufacturers Association sensitivity protocol (30), a peak sensitivity at the center of the FOV of $15 \%$, and a reconstructed spatial resolution of about $3 \mathrm{~mm}$. Importantly, only very modest degradation of spatial resolution due to the high solid-angle coverage and acceptance angles was observed. The system, along with representative images from a low-activity total-body ${ }^{18} \mathrm{~F}$-FDG scan in a nonhuman primate, is shown in Figure 4.

A second mini EXPLORER system based on the exact components that will be used in the human EXPLORER scanner has recently been developed. In addition to being the test environment before scaling up to build the human EXPLORER, this PET/CT system has been designed with companion animal applications in mind and will be installed at the University of California Davis School of Veterinary Medicine.

\section{APPLICATIONS FOR TOTAL-BODY PET}

\section{Human Research}

The major motivation for developing total-body PET is to expand current, and more importantly open new, translational applications for PET in the study of human disease. In addition, the capability of acquiring acceptable images using low administered activities affords opportunities to study normal physiology at radiation doses below those received from a month of natural background radiation, allowing the considerable power of the tracer kinetic principle to be applied, such as in studies of development, aging, nutrition, and exercise. It is anticipated that some of the clinical research areas in which total-body PET methodology is developed and applied may also lead to new

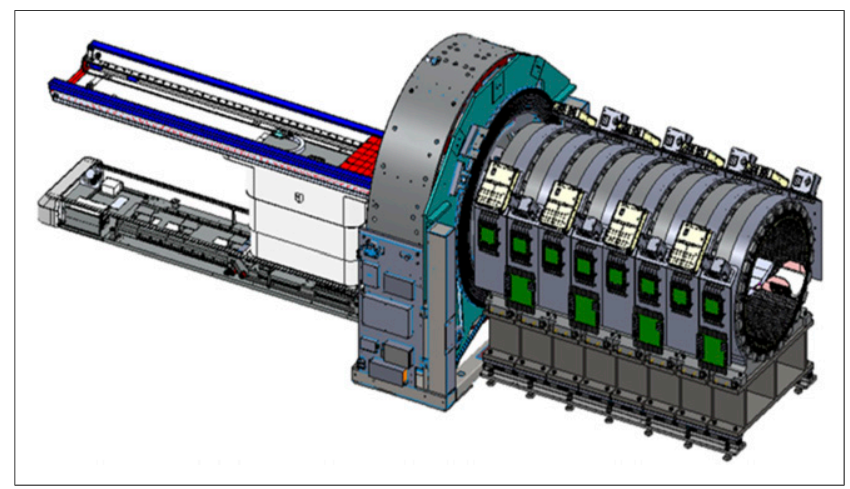

FIGURE 3. Design drawing for EXPLORER PET/CT scanner. (Courtesy of United Imaging Healthcare.) 


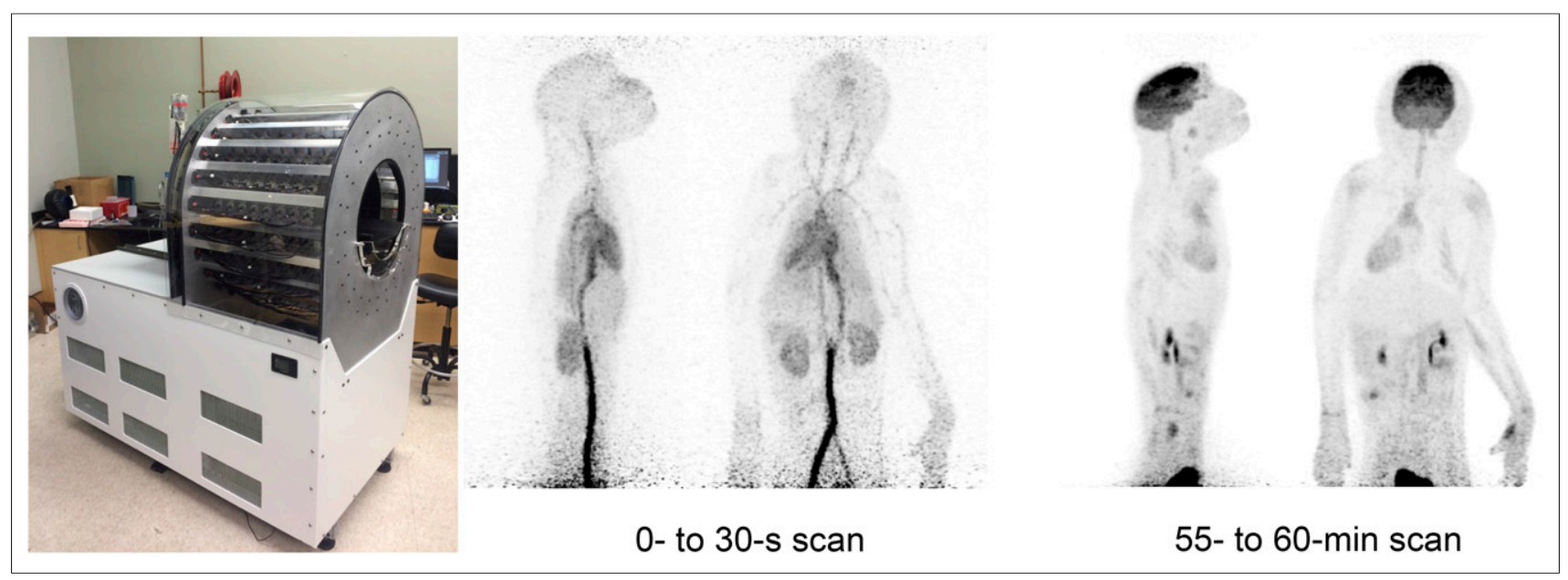

FIGURE 4. Photograph of mini EXPLORER scanner (left) and maximum-intensity-projection images of 2 frames from dynamic total-body imaging study after injection of $8.5 \mathrm{MBq}$ of ${ }^{18} \mathrm{~F}-\mathrm{FDG}(1 / 10$ standard activity) in $4.6-\mathrm{kg}$ rhesus macaque (right).

health-care applications for PET as part of the developing vision of personalized, precision, systems-based medicine (31).

Several significant applications for total-body PET have already been identified through engagement with experts worldwide. We take a moment to briefly expand on several of these below. Undoubtedly, however, there are other opportunities that have been overlooked or not even conceived of at this point. These provide a major motivation for writing this article, as we encourage researchers and clinicians from all disciplines to consider areas in which this sensitive total-body imaging device could help generate new knowledge; improve disease detection, staging, and prognosis; or inform and monitor therapy.

Systemic Disease: Cancer and Beyond. Total-body PET offers several opportunities to change the methodologic approach to cancer detection and staging, and this same methodology could also be applied to other systemic conditions, including inflammation (e.g., sarcoidosis), vascular disease, sepsis, and infectious disease. The increased sensitivity and dynamic range of total-body PET will allow imaging at high SNR at much later times after tracer injection. Tumor contrast typically increases with time as tracers clear from other tissues $(32,33)$; thus, later imaging may reveal a different picture about the extent of disease and allow smaller or less tracer-avid lesions to be seen. A second approach is to use the kinetic information inherent in any total-body PET scan. Even a relatively short (20-30 min) scan will contain considerable kinetic information that might be used to identify small regions of infiltrating tumor cells that are below the resolution limit of the scanner and cannot be seen directly but alter the kinetics within a voxel sufficiently so that their presence can be inferred (34). Such a paradigm could allow low-grade disease (cancer, inflammation, infection) to be detected and quantified. Tracers exist and continue to be developed for cancer and inflammation (35), and although specific tracers for a range of infectious agents (HIV, tuberculosis, malaria, parasites) are sorely needed, some are already in the pipeline (36).

Multiorgan Disease. It has become increasingly apparent that many diseases and conditions thought originally to be confined within a single organ are much more complex and involve the interplay of that organ with other organs or systems (e.g., the endocrine system, the immune system, and the microbiome). An obvious example would be the emerging evidence of the role of the gut-brain axis (which includes the central nervous system, enteric nervous system, immune system, and gut microbiota) in diverse conditions such as anxiety, schizophrenia, autism, and Parkinson disease (37), which classically have been treated as purely the domain of the brain. This link is further reinforced by the fact that cognitive behavioral therapy has been shown to be effective for a range of gastrointestinal disorders, most notably irritable bowel syndrome (38). A second example of multiorgan disease would be the strong link between nonalcoholic fatty liver disease and heart disease, just one of several conditions in which hepatic and cardiac disorders are intertwined (39). In a further example, a clear relationship between the availability of nicotinic acetylcholine receptors in the brain as determined by PET with hepatic nicotine metabolism, and their dependency on smoking, was demonstrated (40). Although these findings lead to an increasing interest in studying and treating disease from a systems perspective, there are few tools and assays that can provide data to support such research. Total-body PET has the necessary capabilities (whole-body coverage, high sensitivity, noninvasiveness, low risk) to permit human research in this area, although new radiotracer development will be needed for many applications, such as to support imaging of endocrine regulation or the mobilization and movement of immune cells to study immune-mediated inflammatory diseases.

Drug Development and Toxicology. The ability to determine the pharmacokinetics of new drugs in all the organs and tissues of the body at low masses and radiation doses has the potential to accelerate translation of new therapeutic agents to humans (41). Early low-risk studies in humans circumvent the many limitations of using animal models to predict drug transport, metabolism, and excretion and could lead to better and earlier go/no-go decisions before launching expensive clinical trials. The same holds true for new imaging radiotracer (or other imaging contrast agent) development, in which pharmacokinetics and human dosimetry could be established using radiation doses that are well below the annual natural background level. The ability to measure slower kinetics relative to the half-life of a given radionuclide across the entire body can be used to improve the precision with which the cumulated activity and thus the absorbed dose are estimated. Another 
area that whole-body pharmacokinetics capability would enable is toxicologic studies. There are many unanswered questions about the fate and the biologic effects of a range of elements, molecules, and particulates found in foods, food packaging, consumer products, and the environment that cannot readily be studied in humans using current techniques.

Monitoring Cellular and Nanoparticle-Based Therapies. Another paradigm in which high sensitivity is generally a prerequisite is imaging studies designed to track the fate of cells (42) or nanoparticles in vivo. With a growing number of clinical trials using therapeutic cells, and large numbers of preclinical studies showing the promise of nanoparticle-mediated therapies, many questions remain as to the distribution and fate of these cells and particles when they are introduced into the body. Radiolabeling these entities (43-45) renders them visible, but with current PET scanners, only large numbers can be detected, and they can be followed for only a relatively short time. Total-body PET has the potential to allow lower numbers of cells and particles to be detected, and after an injection of cells or particles labeled with a long-lived positron emitter such as ${ }^{89} \mathrm{Zr}$ (half-life, $3.3 \mathrm{~d}$ ), it may be possible to follow their fate in vivo for weeks or even a month. Total-body PET could thus be an important tool in measuring and optimizing the efficiency of cell-based therapies, as well as providing information on the delivery and retention of nanoparticles elsewhere in the body over extended time scales. A particular opportunity is to develop the necessary methodology to image the trafficking of immune cells to support the rapid clinical deployment and variable outcomes of cancer immunotherapy.

Maternal-Fetal Medicine. PET has seen virtually no use in human maternal-fetal medicine because of concerns over the effects of radiation on the fetus. However, critical questions exist in maternal-fetal physiology-for example, brain oxygen use, which could be studied using ${ }^{15} \mathrm{O}_{2}$. Other opportunities include the transport of tracers of nutrients across the placenta, fetal inflammation, and infection. The risk due to radiation must be placed in the context of current diagnostic methods that are often invasive and themselves carry significant risk. Earlier work in nonhuman primates with PET $(46,47)$, as well as recent clinical studies in humans with MRI $(48,49)$, demonstrate the value of advanced imaging of the fetus. Total-body PET offers the possibility of conducting scans at extremely low radiation doses, indeed at doses that pregnant women are often exposed to from other sources such as intercontinental flights. Is there a case to be made for ultralow-dose diagnostic PET scanning when the fetus or mother is at risk (50)? How far can the effective dose be reduced while still providing diagnostic information? Total-body PET opens the door to study these challenging questions so that the risk-to-benefit trade-offs and related ethical considerations may be appropriately evaluated.

Normal Physiology and Metabolism. In its early days, PET was used as a research tool (51), with a substantial number of studies being conducted on volunteers, especially to map out task-related activation sites in the brain before the advent of functional MRI, as well as to study a range of physiologic parameters in the brain and heart. It is our hope that with the low-dose capability of a totalbody system, PET will once again become more widely used to study normal biology, metabolism, and physiology, but this time addressing different sets of questions that are not amenable to study with existing tools. New metabolic tracers are becoming available, such as the recent introduction of ${ }^{18} \mathrm{~F}$-fluoroglutamine for imaging of glutamine metabolism (52). There are a broad range of studies that could be conducted to study the role of nutrition and exercise and their impact on metabolism, inflammation, the immune system, the cardiovascular system, the endocrine system, and the microbiome. In addition to established tracers for metabolism and inflammation, new tracers will be needed to assess immune activation and hormonal regulation in vivo.

\section{Clinical Care}

Although the primary driving motivation for developing totalbody PET was originally clinical research, discussions within the community also have highlighted opportunities for improving current clinical care. Taking a standard "eyes-to-thighs" wholebody ${ }^{18} \mathrm{~F}-\mathrm{FDG}$ cancer imaging protocol as a reference, we are anticipating that the first-generation EXPLORER scanner will be able to reduce imaging times by approximately a factor of 24 . Thus, a 12-min multiple-bed-position study could be conducted on a total-body PET scanner in $30 \mathrm{~s}$ in a single bed position with the same image quality. The CT scan would be acquired as the patient moves into the PET scanner, and thus the entire imaging examination could be completed in well under $5 \mathrm{~min}$. Accounting for patient positioning, 40 patients per day (15-min patient time slots, similar to those used for CT examinations) seems entirely feasible for a 10-h working day. Furthermore, images could be obtained, in most patients, in a single breath-hold, reducing motion artifacts and significantly improving lesion detectability in some regions of the body (53).

An alternative approach, recognizing that with a 30 -s acquisition time the throughput is no longer limited by the length of the PET/CT scan but by other factors, would be to use the 24-fold effective sensitivity gain for a more balanced 3-way benefit. One could, for example, reduce absorbed dose by a factor of 2 , reduce imaging time by a factor of 3 , and increase the SNR of the images by a factor of $\sqrt{ } 4=2$. The product of these 3 benefits is still consistent with the 24-fold sensitivity gain. How best to use the sensitivity gain for clinical studies is not clear and will need studies that interrogate not only the diagnostic quality of the resulting images but also the workflow in the hospital and the weighting of radiation dose versus medical benefit. The low-dose procedures point to the opportunity for using PET for selected screening applications, such as a secondary screen for lung cancer after obtaining suggestive low-dose CT results.

A rapidly developing area of clinical application is the use of PET for monitoring radionuclide therapy $(54,55)$, especially for neuroendocrine tumors and prostate cancer. Total-body PET should be an ideal tool for longitudinally evaluating the results of these therapies in multiple metastases spread through the body.

The economics for using a total-body PET/CT scanner in the clinic may at first glance seem daunting, yet a combination of much higher throughput, lower radiation dose, and perhaps improved diagnostic performance (though improved SNR or reduced motion because of shorter scan times) might, in busy centers that currently operate multiple scanners, lead to an acceptable cost-to-benefit ratio. One must also account for the fact that that although the capital cost of a total-body PET/CT scanner will be high, there are other significant factors that determine the ultimate cost of providing a clinical PET/CT service. A further consideration is that distribution of radiotracers becomes more cost-effective if lower injected activities can still yield high-quality images. It may even be possible to distribute tracers based on shorter-lived radionuclides, particularly ${ }^{11} \mathrm{C}$, which could lead to new clinical applications. Overall, there are many forces that ultimately will determine the clinical feasibility 
of total-body PET, including how the cost of the technology evolves, whether multiple vendors compete in offering Food and Drug Administration-approved systems, and an analysis of the benefit versus the cost for specific clinical applications as the axial FOV is increased to $200 \mathrm{~cm}$. Early total-body PET/ CT systems will provide a platform for acquiring data that can start to address the latter question, and time will tell how the other, less predictable, factors will affect the adoption of totalbody PET.

\section{TOWARD FURTHER GAINS IN EFFECTIVE SENSITIVITY}

Although the dramatic increase in geometric efficiency of the EXPLORER scanner will provide a step-change in sensitivity, further significant gains are possible, specifically through improvements in detector efficiency and timing resolution.

\section{Detector Efficiency}

Current PET detectors do not capture $100 \%$ of the radiation incident on them; indeed, the 20-mm-thick lutetium-based scintillators used in many systems can theoretically detect no more than $68 \%$ of the available events, and the number is lower than this because of gaps and dead areas within and between detector modules, as well as rejection of events that deposit only part of their energy in the detectors and fall outside the energy window. However, at best only an approximately 1.5 -fold improvement in sensitivity can be achieved using a combination of detector materials with better photoelectric cross-sections, further reductions in gaps and dead space, and thicker detectors (which will also require good levels of depth encoding so that timing resolution does not deteriorate).

\section{Timing Resolution}

Measuring the difference in arrival time of the two annihilation photons defines, within the uncertainty caused by the finite timing resolution of the detectors and electronics, the location of the decaying nuclide along the line joining the two detectors (Fig. 5). This time-of-flight information spatially constrains the location of the event, leading to improved SNR in the reconstructed image. The relationship is given approximately by

$$
\mathrm{SNR} \propto \frac{1}{\sqrt{\Delta t}}
$$

where $\Delta t$ is the timing resolution. Thus, improving timing resolution from typical values of about 400 ps available today to, for example, $100 \mathrm{ps}$, would lead to a further approximately 2-fold increase in SNR. Since SNR is proportional to the square root of the sensitivity, this SNR increase is equivalent to an approximately 4-fold increase in sensitivity. Laboratory results already have demonstrated timing of under 100 ps under idealized conditions (56), and as sensors, scintillators, and detector designs continue to improve, we can anticipate that the timing performance of scanners will continue to get better. Looking even farther into the future, it may be possible to exploit prompt mechanisms, instead of relying on the relatively slow production of scintillation light, to generate signals in detectors that could obviate image reconstruction and provide event coordinates directly $(57,58)$. This possibility, however, would require a timing resolution on the order of 20 ps to achieve 3-mm spatial resolution.

If future work enables these two additional gains to be combined with the expected 40-fold gain in geometric efficiency of the EXPLORER prototype, then effective gains of over 200-fold with

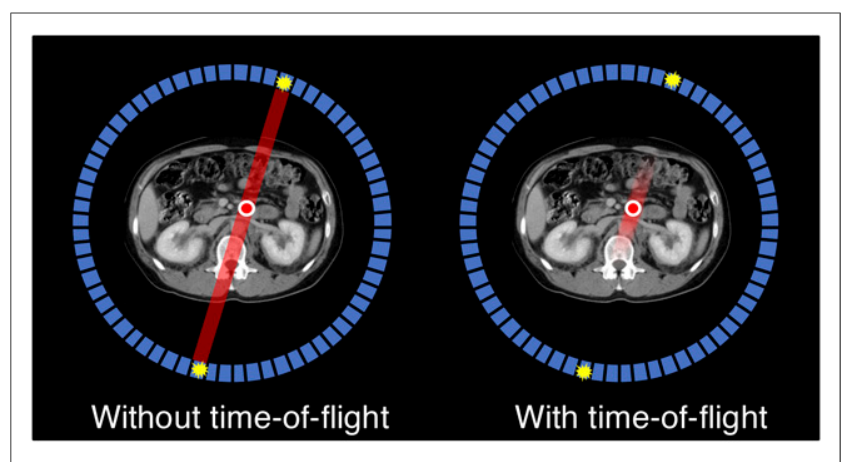

FIGURE 5. Localization through time-of-flight PET showing effect of improving timing resolution. Better timing resolution translates into higher effective sensitivity due to improved localization of each event.

respect to current whole-body PET scanners for total-body imaging are entirely possible. Improvements in timing resolution appear the most promising avenue for further improving the effective sensitivity of PET beyond the geometric gains that will be realized in the EXPLORER prototype. Of course, any gains from improvements in timing resolution will also be beneficial for conventional scanners with a shorter axial FOV.

\section{SUMMARY}

We are hopeful that the large gain in sensitivity of the first EXPLORER total-body PET/CT prototype will open a new chapter in the history of PET development and applications. However, it is a beginning, not an end. Many questions and opportunities need to be addressed. Concerns inevitably focus on the ultimate cost-to-benefit ratio for total-body PET imaging. Careful clinical studies and demonstration of the ability of EXPLORER to generate new knowledge through research will be required to define the benefits. Prospects for cost reduction will focus on decreasing component costs (especially the scintillation crystals, which are a dominant component) and defining the axial extent needed for specific applications. For example, recent demonstrations that bismuth germanate scintillators may be able to support time-of-flight PET $(59,60)$ offer one pathway to lowercost systems without sacrificing sensitivity. As indicated above, opportunities also exist to further improve the effective sensitivity, primarily through better timing resolution. Commercial PET/CT systems have already broken the 400-ps barrier, prototype clinical scanners are closing in on 200-ps timing resolution, and benchtop measurements with latest-generation photodetectors are below 100 ps. For now, the success or otherwise of this endeavor will depend on demonstrating clear-cut benefits in biomedical research and clinical care using the first total-body systems. Some of the possibilities enabled by the large leap in sensitivity and total-body coverage have been outlined in this article; however, we suspect many impactful applications remain to be uncovered and developed. We look forward to working with the PET methodology, clinical research, and health-care communities, as well as industry, to write this next chapter in the history of PET.

\section{DISCLOSURE}

This research was supported by NIH grants R01 CA170874 and R01 CA206187 and by a Research Investment in Science and Engineering Grant from UC Davis. United Imaging Healthcare, 
Philips Healthcare, and Siemens Healthineers contributed time and materials to this project. The authors received travel reimbursement in connection with this research. No other potential conflict of interest relevant to this article was reported.

\section{ACKNOWLEDGMENTS}

The journey to building the first total-body scanner has been a long one, and we gratefully acknowledge the many researchers and clinicians who have engaged with the EXPLORER team and provided advice, suggestions, and support. We particularly acknowledge (arranged alphabetically) Thomas Budinger, Kevin Cox, Michael Graham, Roger Gunn, William Jagust, David Mankoff, Paul Marsden, Steven Meikle, Michael Phelps, Pat Price, Ilan Rabiner, and Richard Wahl. We also are grateful for the input and guidance of our industry advisors at Siemens Healthineers, Philips Healthcare, GE Healthcare, and particularly United Imaging Healthcare. We thank Alice Tarantal for her expertise and assistance with the study shown in Figure 4. Lastly, we thank the following individuals for their major contributions to the EXPLORER program: Martin Judenhofer (project manager), Julien Bec (mechanical engineering), Eric Berg (mini EXPLORER), and Xuezhu Zhang (simulation and image reconstruction).

\section{REFERENCES}

1. Poon JK, Dahlbom ML, Moses WW, et al. Optimal whole-body PET scanner configurations for different volumes of LSO scintillator: a simulation study. Phys Med Biol. 2012;57:4077-4094.

2. Poon JK. The Performance Limits of Long Axial Field of View PET Scanners [thesis]. Davis, CA: University of California, Davis; 2013.

3. Lammertsma AA. Forward to the past: the case for quantitative PET imaging. J Nucl Med. 2017;58:1019-1024.

4. Karakatsanis NA, Lodge MA, Tahari AK, Zhou Y, Wahl RL, Rahmim A. Dynamic whole-body PET parametric imaging: I. Concept, acquisition protocol optimization and clinical application. Phys Med Biol. 2013;58:7391-7418.

5. Ho-Shon K, Feng DG, Hawkins RA, Meikle S, Fulham MJ, Li XJ. Optimized sampling and parameter estimation for quantification in whole body PET. IEEE Trans Biomed Eng. 1996;43:1021-1028.

6. Eriksson L, Townsend DW, Conti M, et al. Potentials for large axial field of view positron camera systems. In: IEEE Nuclear Science Symposium and Medical Imaging Conference (2008 NSS/MIC). Piscataway, NJ: IEEE; 2008: 1632-1636.

7. Couceiro M, Ferreira NC, Fonte P. Sensitivity assessment of wide axial field of view PET systems via Monte Carlo simulations of NEMA-like measurements. Nucl Instrum Meth A. 2007;580:485-488.

8. Cherry SR. The 2006 Henry N. Wagner lecture: of mice and men (and positrons)—advances in PET imaging technology. J Nucl Med. 2006;47: 1735-1745.

9. Crosetto DB. The 3-D complete body screening (3D-CBS) features and implementation. In: IEEE Nuclear Science Symposium and Medical Imaging Conference (2003 NSS/MIC). Piscataway, NJ: IEEE;2004:2415-2419.

10. Badawi RD, Kohlmyer SG, Harrison RL, Vannoy SD, Lewellen TK. The effect of camera geometry on singles flux, scatter fraction and trues and randoms sensitivity for cylindrical 3D PET: a simulation study. IEEE Trans Nucl Sci. 2000;47:1228-1232.

11. Wong WH, Zhang YX, Liu ST, et al. The initial design and feasibility study of an affordable high-resolution 100-cm long PET. In: IEEE Nuclear Science Symposium and Medical Imaging Conference (2007 NSS/MIC). Piscataway, NJ: IEEE; 2007:4117-4122.

12. Watanabe M, Shimizu K, Omura T, et al. A high-throughput whole-body PET scanner using flat panel PS-PMTs. IEEE Trans Nucl Sci. 2004;51:796-800.

13. Surti S, Werner ME, Karp JS. Study of PET scanner designs using clinical metrics to optimize the scanner axial FOV and crystal thickness. Phys Med Biol. 2013;58:3995-4012.

14. MacDonald LR, Harrison RL, Alessio AM, Hunter WCJ, Lewellen TK, Kinahan PE. Effective count rates for PET scanners with reduced and extended axial field of view. Phys Med Biol. 2011;56:3629-3643.
15. Eriksson L, Townsend D, Conti M, et al. An investigation of sensitivity limits in PET scanners. Nucl Instrum Meth A. 2007;580:836-842.

16. Eriksson L, Conti M, Melcher CL, et al. Towards sub-minute PET examination times. IEEE Trans Nucl Sci. 2011;58:76-81.

17. Surti S, Karp JS. Impact of detector design on imaging performance of a long axial field-of-view, whole-body PET scanner. Phys Med Biol. 2015;60:53435358 .

18. Schmall JP, Karp JS, Werner M, Surti S. Parallax error in long-axial field-of-view PET scanners: a simulation study. Phys Med Biol. 2016;61:5443-5455.

19. Wang W, Gagnon D, Hiu X, Wang W, et al., inventors; Toshiba Medical Systems Corporation, assignee. Field-of-view-dependent coincidence window for positron emission tomography. U.S. patent US8809792 B2. August 19, 2014.

20. Kalender WA. Dose in x-ray computed tomography. Phys Med Biol. 2014;59: R129-R150.

21. Rothfuss H, Panin V, Moor A, et al. LSO background radiation as a transmission source using time of flight. Phys Med Biol. 2014;59:5483-5500.

22. Mollet P, Keereman V, Vandenberghe S. Experimental evaluation of simultaneous emission and transmission imaging using TOF information. In: IEEE Nuclear Science Symposium and Medical Imaging Conference (2011 NSS/ MIC). Piscataway, NJ: IEEE;2011:2976-2980.

23. Attariwala R, Picker W. Whole body MRI: improved lesion detection and characterization with diffusion weighted techniques. J Magn Reson Imaging. 2013; 38:253-268.

24. Home page. EXPLORER consortium website. https://explorer.ucdavis.edu/. Accessed October 26, 2017.

25. High-risk, high-reward research program. National Institutes of Health website. https://commonfund.nih.gov/highrisk. Reviewed October 11, 2017. Accessed October 26, 2017.

26. Jakoby BW, Bercier Y, Conti M, Casey ME, Bendriem B, Townsend DW. Physical and clinical performance of the $\mathrm{mCT}$ time-of-flight PET/CT scanner. Phys Med Biol. 2011;56:2375-2389.

27. Somlai-Schweiger I, Schneider FR, Ziegler SI. Performance analysis of digital silicon photomultipliers for PET. J Instrum. 2015;10:P05005.

28. Degenhardt C, Rodrigues P, Trindade A, et al. Performance evaluation of a prototype positron emission tomography scanner using digital photon counters (DPC). In: IEEE Nuclear Science Symposium and Medical Imaging Conference Record (2012 NSS/MIC). Piscataway, NJ: IEEE;2012:2820-2824.

29. Miller M, Zhang J, Binzel K, et al. Characterization of the Vereos digital photon counting PET system [abstract]. J Nucl Med. 2015;56(suppl 3):434.

30. NEMA NU 2-2012 Performance Measurements of Positron Emission Tomographs. Arlington, VA: National Electrical Manufacturers Association; 2013.

31. Cherry SR, Badawi RD, Karp JS, Moses WW, Price P, Jones T. Total-body imaging: transforming the role of positron emission tomography. Sci Transl Med. 2017;9:eaaf6169.

32. Kubota K, Itoh M, Ozaki K, et al. Advantage of delayed whole-body FDG-PET imaging for tumour detection. Eur J Nucl Med. 2001;28:696-703.

33. Lodge MA, Lucas JD, Marsden PK, Cronin BF, O'Doherty MJ, Smith MA. A PET study of ${ }^{18}$ FDG uptake in soft tissue masses. Eur J Nucl Med. 1999;26:2230.

34. Price PM, Badawi RD, Cherry SR, Jones T. Ultra staging to unmask the prescribing of adjuvant therapy in cancer patients: the future opportunity to image micrometastases using total-body ${ }^{18}$ F-FDG PET scanning. J Nucl Med. 2014; 55:696-697.

35. Wu C, Li F, Niu G, Chen XY. PET imaging of inflammation biomarkers. Theranostics. 2013;3:448-466.

36. Santangelo PJ, Rogers KA, Zurla C, et al. Whole-body immunoPET reveals active SIV dynamics in viremic and antiretroviral therapy-treated macaques. Nat Methods. 2015;12:427-432.

37. Klingelhoefer L, Reichmann H. Pathogenesis of Parkinson disease: the gut-brain axis and environmental factors. Nat Rev Neurol. 2015;11:625-636.

38. Ford AC, Quigley EMM, Lacy BE, et al. Effect of antidepressants and psychological therapies, including hypnotherapy, in irritable bowel syndrome: systematic review and meta-analysis. Am J Gastroenterol. 2014;109:1350-1365.

39. Møller S, Bernardi M. Interactions of the heart and the liver. Eur Heart J. 2013;34:2804-2811.

40. Dubroff JG, Doot RK, Falcone M, et al. Decreased nicotinic receptor availability in smokers with slow rates of nicotine metabolism. J Nucl Med. 2015;56:17241729 .

41. Bergstrom M. The use of microdosing in the drug development of small organic and protein therapeutics. J Nucl Med. 2017;58:1188-1195.

42. Kircher MF, Gambhir SS, Grimm J. Noninvasive cell-tracking methods. Nat Rev Clin Oncol. 2011;8:677-688.

43. Adonai $\mathrm{N}$, Nguyen $\mathrm{KN}$, Walsh $\mathrm{J}$, et al. Ex vivo cell labeling with $\mathrm{Cu}-64$ pyruvaldehyde-bis(N-4-methylthiosemicarbazone) for imaging cell trafficking 
in mice with positron-emission tomography. Proc Natl Acad Sci USA. 2002;99:3030-3035.

44. Bansal A, Pandey MK, Demirhan YE, et al. Novel Zr-89 cell labeling approach for PET-based cell trafficking studies. EJNMMI Res. 2015;5:19.

45. Charoenphun P, Meszaros LK, Chuamsaamarkkee K, et al. $\left.{ }^{89} \mathrm{Zr}\right]$ oxinate $_{4}$ for long-term in vivo cell tracking by positron emission tomography. Eur J Nucl Med Mol Imaging. 2015;42:278-287.

46. Eyal S, Chung FS, Muzi M, et al. Simultaneous PET imaging of P-glycoprotein inhibition in multiple tissues in the pregnant nonhuman primate. $\mathrm{J} \mathrm{Nucl} \mathrm{Med}$. 2009;50:798-806.

47. Berglund L, Halldin C, Lilja A, et al. C-11 methionine kinetics in pregnant rhesus-monkeys studied by positron emission tomography: a new approach to fetomaternal metabolism. Acta Obstet Gynecol Scand. 1984;63:641645.

48. Glenn OA. MR imaging of the fetal brain. Pediatr Radiol. 2010;40:68-81.

49. Griffiths PD, Bradburn M, Campbell MJ, et al. Use of MRI in the diagnosis of fetal brain abnormalities in utero (MERIDIAN): a multicentre, prospective cohort study. Lancet. 2017;389:538-546.

50. Jones T, Budinger TF. The potential for low-dose functional studies in maternalfetal medicine using PET/MR imaging. J Nucl Med. 2013;54:2016-2017.

51. Phelps ME. PET: Molecular Imaging and Its Biological Applications. New York, NY: Springer; 2004.
52. Zhu L, Ploessl K, Zhou R, Mankoff D, Kung HF. Metabolic imaging of glutamine in cancer. J Nucl Med. 2017;58:533-537.

53. Nehmeh SA, Erdi YE, Meirelles GSP, et al. Deep-inspiration breath-hold PET/ CT of the thorax. J Nucl Med. 2007;48:22-26.

54. Baum RP, Kulkarni HR. THERANOSTICS: from molecular imaging using Ga68 labeled tracers and PET/CT to personalized radionuclide therapy - the Bad Berka experience. Theranostics. 2012;2:437-447.

55. Jadvar H. Targeted radionuclide therapy: an evolution toward precision cancer treatment. AJR. 2017;209:277-288.

56. Gundacker S, Acerbi F, Auffray E, et al. State of the art timing in TOF-PET detectors with LuAG, GAGG and L(Y)SO scintillators of various sizes coupled to FBK-SiPMs. J Instrum. 2016;11:P08008.

57. Gundacker S, Auffray E, Pauwels K, Lecoq P. Measurement of intrinsic rise times for various $\mathrm{L}(\mathrm{Y}) \mathrm{SO}$ and LuAG scintillators with a general study of prompt photons to achieve $10 \mathrm{ps}$ in TOF-PET. Phys Med Biol. 2016;61:2802-2837.

58. Lecoq P, Korzhik M, Vasiliev A. Can transient phenomena help improving time resolution in scintillators? IEEE Trans Nucl Sci. 2014;61:229-234.

59. Brunner SE, Schaart DR. BGO as a hybrid scintillator/Cherenkov radiator for cost-effective time-of-flight PET. Phys Med Biol. 2017;62:4421-4439.

60. Kwon SI, Gola A, Ferri A, Piemonte C, Cherry SR. Bismuth germanate coupled to near ultraviolet silicon photomultipliers for time-of-flight PET. Phys Med Biol. 2016;61:L38-L47. 